\title{
Proposta de uma Arquitetura de Agentes Móveis para Recuperação de Informação Útil e Distribuída no Processo de Gestão do Conhecimento
}

\author{
Andréa Sabedra Bordin ${ }^{1}$, Renato Balancieri ${ }^{1}$ \\ ${ }^{1}$ Programa de Pós-Graduação em Engenharia e Gestão do Conhecimento, Universidade Federal de Santa \\ Catarina, Trindade, Florianópolis, SC, Brasil \\ \{abordin, renato\}@stela.ufsc.br
}

\begin{abstract}
Resumo - O processo de gestão do conhecimento no contexto institucional caracteriza-se pela gestão da competência dos seus colaboradores, seus relacionamentos internos e externos. Nesse sentido, abordagens e técnicas computacionais que capturem informações sobre as competências do colaborador de maneira explícita e tácita fazem-se necessárias. A abordagem utilizada neste artigo considera que na maioria das situações o colaborador explicita suas competências na forma de um curriculum vitae, o qual contém informações sobre sua formação escolar, experiência de trabalho, suas aptidões, etc. e o armazena dentro do seu computador pessoal no formato textual. Diante disso, propõe-se a utilização de uma arquitetura de agentes móveis que realize a detecção dessas informações na intranet institucional para formação de uma base textual única e centralizada que será utilizada no processo de gestão do conhecimento.
\end{abstract}

Palavras-chave: Gestão do Conhecimento, Recuperação de Informação, Agentes Móveis.

Abstract - Within the institutional context, the competence management of the institution's collaborators and its external and internal relationships characterize the knowledge management process. Thus, computational approaches and techniques that capture information about the collaborator's abilities, in an explicit and approximate away, are necessary. The approach used in the present article considers that, in most situations, the collaborator makes explicit his/her abilities in a curriculum vita, which contains information about his/her education, working experience, aptitude and so forth, and then stores it in a personal computer in a textual format. In light of this, it is proposed the employment of a mobile agents architecture that is able to detect these pieces of information in the institutional Intranet in order to form a unique and centralized textual base, which will then be used in the knowledge management process.

Keywords: Knowledge Management, Information Recovery, Mobile Agent.

\section{Introdução}

No contexto institucional é cada vez mais freqüente a utilização de um computador pessoal interconectado em rede como ferramenta de trabalho. Esse computador acaba sendo o local em que os colaboradores armazenam em arquivos suas informações de trabalho e informações pessoais, e, entre essas, 0 arquivo com suas informações curriculares. Essa situação é dinâmica, ou seja, as informações curriculares podem ser constantemente atualizadas pelos usuários de cada computador e novos usuários podem se conectar à rede institucional. Portanto, saber onde essa informação está e monitorar sua atualização torna-se um procedimento necessário quando o objetivo é utilizála no processo de gestão do conhecimento.

Nesse processo, o conhecimento é um dos recursos fundamentais para as organizações, portanto, propor mecanismos que o identifiquem e o gerenciem é estratégico.

Diante desse panorama, o objetivo principal do artigo é propor uma solução que utilize uma arquitetura de agentes móveis para gerenciar a detecção e posterior busca de informação especializada e útil para o processo de gestão do conhecimento. Com as informações reunidas num mesmo repositório será possível, por exemplo, traçar o perfil de cada colaborador, detectar áreas de excelência e lacunas de competências a serem preenchidas, enfim, possibilitar a tomada de decisão para um melhor gerenciamento de competências individuais e, conseqüentemente, do conhecimento.

A proposta utilizará uma arquitetura de agentes móveis na qual os agentes serão disparados periodicamente de uma estação central para todas as estações da intranet da instituição e aplicarão técnicas de recuperação de informação para descobrir documentos relevantes, nesse caso, que contenham informações curriculares. $O$ agente 
armazenará informações como nome, data e localização dentro da rede dos documentos selecionados e, ao final do processo, retornará ao ponto de origem com essas informações, as quais serão armazenadas em uma base de dados e utilizadas em processos futuros que fazem parte da solução proposta (explicada na Seção 4).

O artigo está estruturado em cinco seções: as Seções 2 e 3 abordam os tópicos relativos à gestão do conhecimento e aos agentes móveis, respectivamente; a Seção 4 apresenta o detalhamento da proposta; e, finalmente, a Seção 5 contém as conclusões da realização deste trabalho.

\section{Gestão do Conhecimento}

Atualmente estamos vivenciando a chamada "Era do Conhecimento" e, como conseqüência disso, a "economia do conhecimento", em que as empresas e as nações dependem de sua capacidade de gerar, processar e aplicar de forma eficiente o conhecimento. Como resultado, modelos de gestão do conhecimento são cada vez mais necessários.

Segundo Sveiby [1], a gestão do conhecimento não é mais uma moda de eficiência operacional, ela já faz parte da estratégia empresarial. Nonaka e Takeuchi [2], afirmam que, para se tornar uma empresa que gera conhecimento (knowledge creating company), a organização deve completar uma espiral do conhecimento, espiral que vai de tácito a tácito, de explícito a explícito, de tácito a explícito e, finalmente, de explícito a tácito. Logo, o conhecimento deve ser articulado e então internalizado para tornar-se parte da base de conhecimento de cada pessoa.

Gestão do conhecimento é, portanto, o processo sistemático de identificação, criação, renovação e aplicação dos conhecimentos que são estratégicos na vida de uma organização; é a administração dos ativos de conhecimento das organizações. A gestão do conhecimento permite à organização saber o que ela sabe.

Os principais objetivos da gestão do conhecimento organizacional são melhorar a produtividade do conhecimento dos trabalhadores da organização e proporcionar meios para a rápida construção e utilização da coleção do conhecimento da organização.

A gestão do conhecimento dá importância principalmente às pessoas, a seus trabalhos práticos e culturais, e também decide como e quais tecnologias deverão ser empregadas nesse cenário.

De acordo com Neto [3], uma visão global de como o ambiente do conhecimento está estruturado pode ser expressa através da descrição de uma estrutura esquemática baseada no conhecimento. Tal estrutura de gestão do conhecimento possui uma arquitetura baseada em uma capacidade social e tecnológica para produzir repositórios que possam possibilitar comunicação do conhecimento dos trabalhadores por toda a organização. São necessários sistemas de navegação, ferramentas e também capacidade de proporcionar a criação de um fluxo de conhecimento pela organização.

Segundo a proposta de Borghoff e Pareschi [4], a arquitetura de gestão do conhecimento é composta de quatro componentes: fluxo do conhecimento, cartografia do conhecimento, comunidade dos trabalhadores do conhecimento e repositório e biblioteca de conhecimento. A Figura 1 ilustra a idéia da estrutura da arquitetura, em que o componente fluxo do conhecimento liga os outros três componentes. Esse esquema pode ser visto de duas maneiras: a) primeiramente, como uma extensão teórica da noção de conhecimento de Nonaka e Takeuchi [2], adaptada para suportar a tecnologia da informação; b) por outro lado, o esquema é firmemente enraizado em solo empírico e tem sido comprovado e testado em algumas organizações, tais como a Xerox, a IBM e através de estudo de caso e entrevistas em várias organizações. 


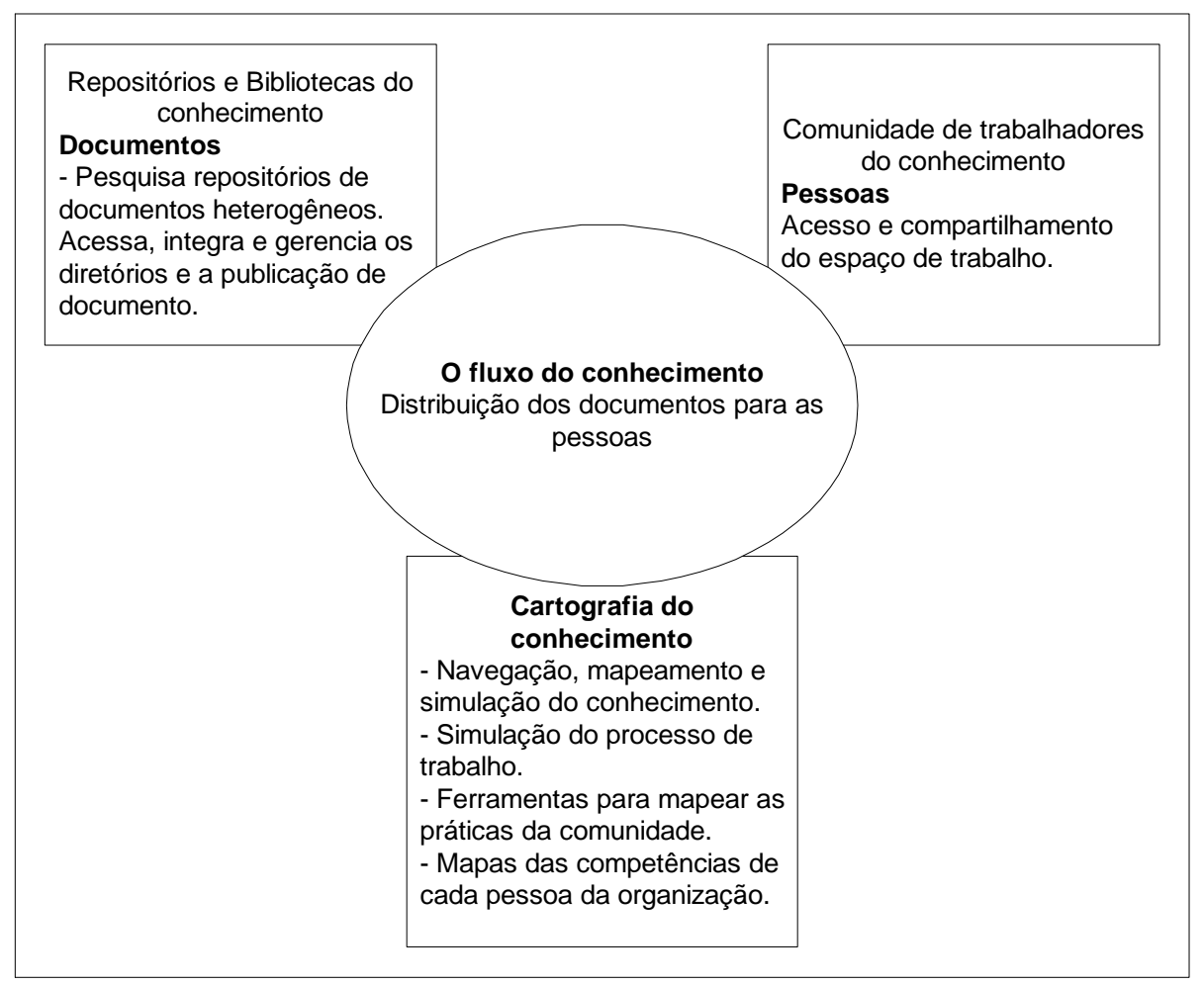

Figura 1 - Estrutura do Gerenciamento do Conhecimento (Adaptado de: BORGHOFF; PARESCHI [4])

Como pode ser visto na Figura 1, o processo de gestão do conhecimento perpassa pela gestão de competências dentro da organização. Segundo Torres e Torres [5], competência é fazer uso do conhecimento para executar ações. É importante destacar que não há um consenso em relação à maneira como competências são formadas. Alguns autores debatem esse tema, tais como Zarifian [6], Fleury e Fleury [7] e Prahalad e Hamel [8]. Luz [9] menciona duas linhas de definições principais: a) a escola francesa, que enfatiza a vinculação entre trabalho e educação, considerando as competências como resultado da educação sistemática e valorizando o modo como as escolas enriquecem o repertório de habilidades dos alunos; e b) a escola britânica, que define competências tomando como referência o mercado de trabalho e enfatiza fatores ou aspectos ligados à descrição de desempenhos requeridos pelas organizações produtivas.

Independentes de sua definição, competências pessoais devem ser capazes de agregar valor ao patrimônio de conhecimentos da organização, com isso modelos e sistemas capazes de gerenciar competências se fazem necessários e estão incorporados à questão da gestão do conhecimento organizacional.

Portanto, o desenvolvimento de uma ferramenta para mapear e catalogar as competências de cada pessoa da organização torna-se cada vez mais essencial. Segundo Borghoff e Pareschi [4], esse tipo de ferramenta caracteriza as atividades do conhecimento do trabalhador em atividades multipessoais localizadas em um espaço de trabalho, ou também denominado "trabalho ecológico". Este trabalho pode ser descrito em relação ao espaço fisicamente ocupado pelo conhecimento do trabalhador, aos relacionamentos sociais, aos trabalhos práticos, etc.

\section{Agentes Móveis}

\subsection{Definições e principais características}

Um agente móvel é uma entidade de software independente que age em nome de um usuário para realizar uma determinada tarefa, interagindo e cooperando com o seu ambiente e com outros agentes [10].

Agentes móveis são utilizados em sistemas em rede com o objetivo de facilitar o controle e a realização de tarefas distribuídas e coordenadas entre indivíduos e sistemas. O fundamento principal está na capacidade de transferir o seu programa ou o código de execução de um ponto a outro da rede e reiniciar ou continuar sua execução nesse novo ponto. $O$ agente também pode armazenar informações e transferi-las com o seu código.

O conceito de agentes móveis tem sido 
proposto para suportar diferentes tipos de aplicações, entre elas comércio eletrônico [11]; gerenciamento de workflows [12]; gerenciamento de redes [13]; e implementação de serviços de telecomunicação e recuperação de informações distribuídas [14].

No contexto de busca e recuperação de informações distribuídas é necessário programar o agente com algoritmos complexos de busca e recuperação de informação, e despachá-los para os locais onde se encontram as informações.

Existe um esforço recente de padronização das características de um agente móvel que é a norma MASIF (Mobile Agent System Interoperability Facilities), proposta pelo OMG (Object Manager Group)[15], a qual define um conjunto de interfaces básicas que esse sistema deve possuir.

Pode-se resumir o conjunto de características encontradas na literatura [16, 17] como:

Autonomia: ações executadas pelos agentes dependem exclusivamente das informações por eles obtidas e dos seus padrões de decisão.

Movimento: constitui a transferência dos dados, do código e do estado de execução de um lugar para outro.

Identidade: a navegação e multiplicação dos agentes ao longo de uma rede tornam essencial que eles se diferenciem uns dos outros, embora existam aplicações nas quais eles possuem identificação única. Nesse caso, a ênfase é dada na execução das atividades em paralelo. A identificação do agente geralmente fica associada ao ponto de rede em que foi criado.

Ciclo de Vida: diz respeito ao processo de criação, tempo de vida e término do agente. Este é criado quando uma requisição é feita pelo usuário ou por outro agente a uma plataforma de agentes.

Reprodução: agentes podem criar outros passando adiante o seu próprio "código genético".

Execução de Serviços: o algoritmo de execução de um agente pode ser classificado quanto à sua estrutura e à sua forma de operação. Quanto à estrutura, ele pode ter três: proprietária, carregada e nativa. Quanto à forma de operação, pode ser reativo, espontâneo ou proativo.

Comunicação: tem a finalidade de trocar dados e informações e coordenar a ação entre agentes ou grupos de agentes. É definida pela sua forma de endereçamento, transmissão e conexão e linguagem das mensagens trocadas.

\subsection{Arquitetura}

A arquitetura de um sistema de agentes móveis é constituída pela sua plataforma de operação e pelos agentes que rodam [18] sobre si.

As plataformas são os locais, places, ou nós da rede por onde os agentes irão passar e serão executados, e devem estar instaladas em localizações identificadas.

Existem várias plataformas comerciais ou acadêmicas que dão suporte à criação ou à programação de agentes móveis [19, 20, 16, 21], com funcionalidades, dependendo do foco principal de aplicação. As mais recentes são desenvolvidas na linguagem Java, o que permite que sejam executadas em diferentes plataformas.

A Figura 2 ilustra a arquitetura utilizada pela plataforma Aglets, desenvolvida em Java pela IBM, a qual é muito utilizada em aplicações de rede, como comércio eletrônico, gerenciamento e busca de informação, e infra-estrutura de rede.

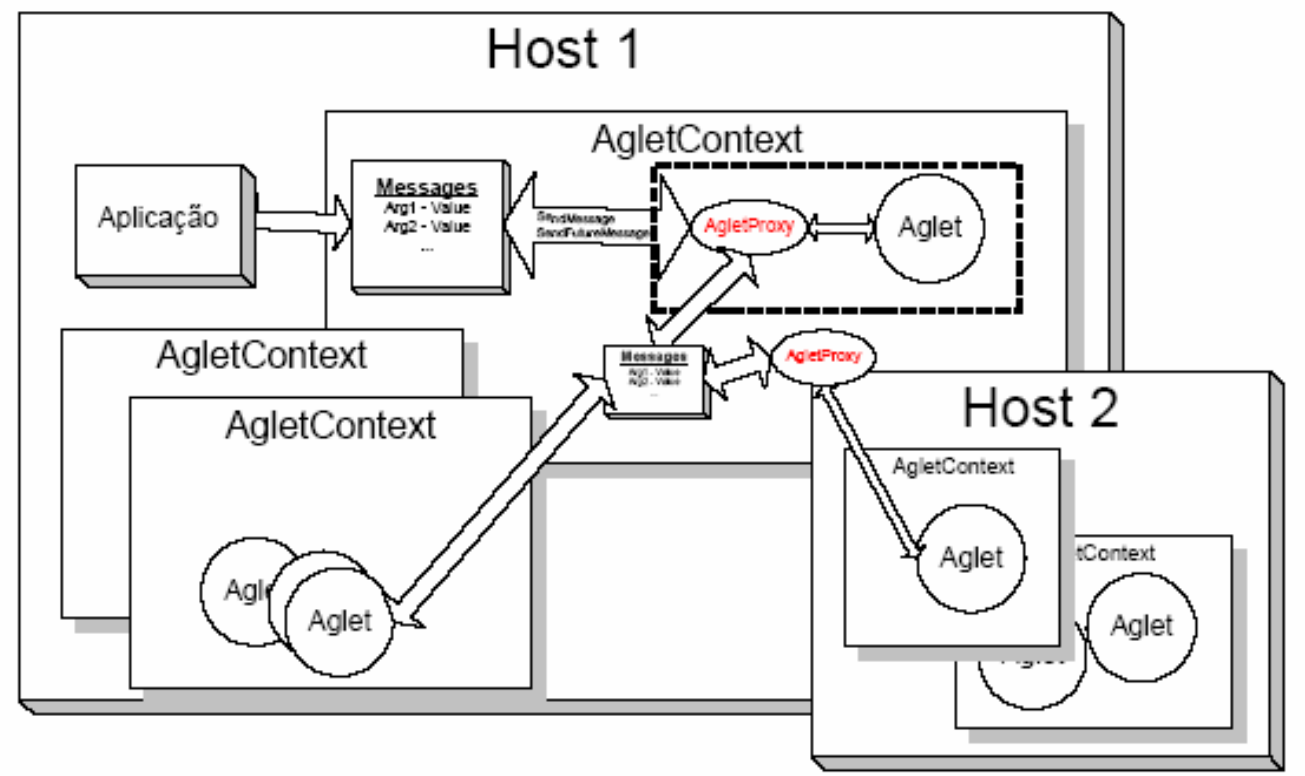

Figura 2 - Arquitetura dos Aglets [22] 
Nessa plataforma o Host representa o local físico da rede, os AgletContexts são locais virtuais que podem ser criados dentro do mesmo nó físico. As mensagens entre os agentes são feitas diretamente entre eles por objetos AgletProxies, os quais permitem comunicação dos agentes dentro do mesmo local da rede ou remotamente.

\subsection{Formas de Operação}

Segundo Kendal et al. [23], as formas de operação dos agentes podem ser divididas em várias categorias, são elas:

? forma de ataque: solitário, quando o agente é programado e migra por todos os pontos da rede executando uma tarefa ou em bandos, quando são enviados vários agentes concomitantemente para vários locais para realização de uma mesma tarefa.

? forma de movimentação: alfândega, o agente deve possuir uma espécie de registro para usufruir do local; trilha, são definidos preliminarmente os percursos para atingir o destino; sinais, não se sabe previamente o trajeto, mas cada um é redirecionado para cada novo ponto ao se chegar nas marcas específicas.

? forma de cooperação: não cooperativa, os agentes não trocam informações entre si; compartilhada, trocam informações com outros na intenção de executar determinada tarefa; controlada, quando se têm certos agentes que agem como gerentes de grupo e controlam as tarefas executadas por um grupo.

? forma de aprendizado: processo em que o agente processa as informações obtidas ao longo de seu ciclo de vida, adaptando seu curso de ação em função das novas informações.

\section{Detalhamento da Proposta}

A solução proposta neste artigo utilizará uma arquitetura de agentes móveis e terá o objetivo geral de pesquisar informações sobre fontes de dados textuais que armazenam informações curriculares que estejam em qualquer uma das estações de uma intranet institucional. As informações sobre os documentos pesquisados que os agentes retornarão serão utilizadas no processo, que posteriormente solicitará o envio desses documentos pelos seus proprietários para um determinado departamento ou pessoa dentro da instituição. Os documentos enviados farão parte de uma base textual com informações curriculares sobre os colaboradores.

As informações sobre competências contidas nessa base textual poderão auxiliar no processo de gestão do conhecimento, na medida em que ali serão encontradas informações que caracterizam o perfil de cada colaborador, permitindo uma tomada de decisão para um melhor gerenciamento de competência e conseqüentemente do conhecimento.

A pesquisa por informações, como, por exemplo, competências específicas nessa base textual, poderá ocorrer documento a documento, ou poderá ser otimizada se forem aplicadas técnicas de recuperação de informação e text mining.

A principal justificativa para a escolha de uma arquitetura de agentes móveis está ligada ao próprio conceito de agente, que é uma entidade capaz de executar tarefas pelo usuário. Nessa proposta isso se concretiza visto que o usuário não irá se preocupar em buscar essas informações na própria máquina, já que o agente fará isso por ele.

Outra justificativa diz respeito à diminuição de tráfego na rede. Agentes móveis podem empacotar a conversação inteira em uma única transmissão de agente, que pode reduzir 0 tráfego de rede sensivelmente Além disso, o custo de desenvolvimento e manutenção de agentes móveis é menor em relação a agentes estáticos.

A arquitetura fará uso da plataforma Aglets, devido ao seu intensivo uso no contexto de aplicações de rede. A forma de operação dos agentes, segundo definições da seção anterior, caracteriza-se como forma de ataque em bandos, forma de movimentação em trilha, forma de cooperação não cooperativa.

A metodologia de desenvolvimento desta proposta seguirá os passos descritos abaixo.

a) Descoberta das estações de trabalho ativas na intranet institucional - criação de uma aplicação na linguagem Java para realizar um "ping" na faixa de endereços de rede passados pelo administrador da rede.

b) Envio dos agentes responsáveis por descobrir a informação às estações ativas - os agentes serão criados e enviados para os endereços ativos da rede, conforme a resposta da execução anterior. É importante destacar que essa plataforma que dá suporte à utilização de agentes móveis, também conhecida como agência, deverá estar instalada em todas as estações.

c) Execução da tarefa pelos agentes cada agente numa estação de trabalho terá o objetivo de verificar todos os documentos do tipo não estruturado, ou seja, textuais, e armazenar informações sobre aqueles que atendam ao critério de busca. Esse processo dar-se-á através de técnicas de recuperação de informação que não serão abordadas de maneira detalhada neste artigo. 
Uma técnica simples e possível seria pesquisar todos os arquivos-texto que contenham algumas palavras-chave, como currículo, formação, cursos, escolaridade, etc.

Após o término da tarefa, cada agente voltará ao seu ponto de partida com informações curriculares desejadas sobre os arquivos encontrados:: nome do arquivo, localização e data de atualização.

Essas informações serão armazenadas em uma base de dados na estação de trabalho que disparou o processo de criação e envio dos agentes e serão úteis nos próximos passos. A Figura 3 ilustra esses três primeiros passos.

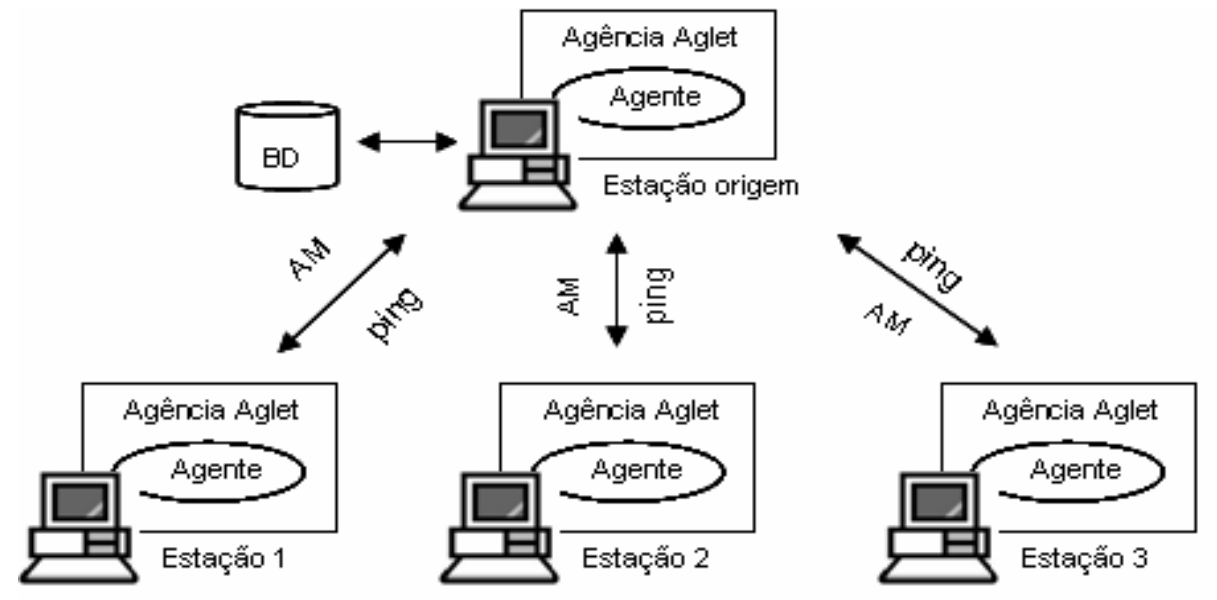

Figura 3 - Arquitetura de agentes móveis para recuperação de informação

A intenção é executar esse processo com uma periodicamente média, pois informações curriculares não são atualizadas com freqüência. No intuito de facilitar a pesquisa nas execuções seguintes do processo de envio dos agentes, a investigação não será realizada em todos os documentos da estação, ou seja, cada agente levará consigo as informações sobre os documentos encontrados na pesquisa anterior em determinada estação de trabalho. Por exemplo, ao pesquisar novamente a estação 2, o agente levará consigo as informações da pesquisa anterior na estação 2; isso fará com que o agente pesquise somente o(s) documentos(s) específico(s) e só atualize as informações quando houver diferenças na data de atualização do arquivo. No entanto, outros documentos podem ser criados nessa estação. Por causa disso, o algoritmo de pesquisa do agente deverá ser programado para executar pesquisa em toda a base de documentos também periodicamente.

d) Solicitação do envio dos documentos - de posse da informação sobre os documentos encontrados nas estações, uma aplicação solicitará automaticamente aos usuários proprietários o envio dos documentos via e-mail.

e) Recepção dos documentos - os documentos serão recebidos através de e-mail e armazenados, formando uma base de dados textual com subsídios para pesquisa de informações sobre competências dos colaboradores.

\section{Conclusão}

Neste trabalho foram introduzidos conceitos básicos de gestão do conhecimento e logo após conceitos fundamentais para compreender a estrutura do gerenciamento do conhecimento com apoio da tecnologia. Apresentou-se uma fundamentação sobre a técnica de agentes móveis e em seguida foi detalhada a solução que utiliza essa abordagem.

Com base na pesquisa realizada conclui-se que o uso de agentes móveis para recuperação de informação distribuída e útil no processo de gerenciamento do conhecimento é uma abordagem factível. Além disso, no contexto empregado esses agentes tendem a ser muito importantes para as organizações, visto que podem ajudar a construção de um repositório com as competências dos colaboradores institucionais, facilitando, assim, a busca por informações que propiciem a criação de novos conhecimentos individuais e organizacionais, tornando a organização mais competitiva.

Como trabalhos futuros é pretendido o aprofundamento nas pesquisas sobre as técnicas de recuperação de informação e técnicas de text mining, sendo esta de extrema relevância no processo de mineração de informações em uma base de dados textual. 


\section{Referências}

1. Sveiby, K. E.: A nova riqueza das organizações. Campus Rio de Janeiro (1998)

2. Nonaka, I., Takeuchi, H.: Criação do conhecimento na empresa. Campus Rio de Janeiro (1997)

3. Neto, J. B.: Tecnologia da Informação para o Gerenciamento do Conhecimento obtido das bases de dados de uma Organização. Dissertação (Mestrado em Engenharia de Produção) - Florianópolis (2001)

4. Borghoff, U., Pareschi, R.: Information technology for knowledge management. Springer-Verlag Germany (1998)

5. Torres, D. M., Torres, M. M.: Árvore de Competências. Projeto final em Gestão do conhecimento e inteligência empresarial. COPPE-UFRJ (2002)

6. Zarifian, P.: Objetivo competência: por uma nova lógica. Atlas São Paulo (2001)

7. Fleury, A., Fleury, M. T. L.: Estratégias empresariais e formação de competências: um quebra-cabeça caleidoscópico da indústria brasileira. Atlas São Paulo (2001)

8. Prahalad, C. K., Hamel, G.: The core competence of the corporation. Harvard Business Review, Vol. 68, n. 3 (1990) 79-91

9. Luz, T. R.: Telemar-Minas: Competências que marcam a diferença. Tese (Doutorado em Administração) - CEPEAD-UFMG Belo Horizonte (2001)

10. Karmouch, A., Pham, V. A.: Mobile Software Agents: An Overview. IEEE Communications Magazine (1998)

11. Chrysanthis P. K. et al.: Establishing Virtual Enterprises by means of Mobile Agents. In: RESEARCH ISSUES IN DATA ENGINEERING WORKSHOP, Proceedings... Sydney,Australia (1999)

12. Cabri G., Leonardi L., Zambonelli F.: A Web Infrastructure for People and Agent Interaction and Collaboration. In: IEEE NINTH INTERNATIONAL WORKSHOPS ON ENABLING TECHNOLOGIES: INFRASTRUCTURE FOR COLLABORATIVE ENTERPRISES (WETICE), NIST, USA (2000)

13. Rubinstein M. G., Duarte, O. C. M. B.: Análise da Eficiência de Agentes Móveis no Gerenciamento de Redes. In: XIX CONGRESSO NACIONAL DA SOCIEDADE BRASILEIRA DE COMPUTAÇÃO, XXVI SEMINÁRIO INTEGRADO DE SOFTWARE E HARDWARE - SEMISH'99, 1. Rio de Janeiro, July (1999) 167-178

14. Radtke P. V. W., Kaestner, A.: MOTHRA: Uma Proposta de Arquitetura Baseada em Agentes
Móveis para Recuperação de Textos e Hiperdocumentos. In: ISDM' 98, Curitiba (1998)

15. Object Management Group. Mobile Agent System Interoperability Facilities-MASIF (1997)

16. Peine H., Stolpmann, T.: The Architecture of ARA Plataform for Móbile Agents. In: FIRST INTERNATIONAL WORKSHOP ON MOBILE AGENTS 97 (MA'97), Berlin, Germany (1997) 7-8

17. Franklin S., Graesser, A.: Is it an Agent, or just a Program?:A Taxonomy for Autonomous Agents. In: THIRD INTERNATIONAL WORKSHOP ON AGENT THEORIES, ARCHITECTURES AND LANGUAGES (1996)

18. Braga, A. L.: Utilização de Agentes Móveis em Recuperação e Troca de Dados. Exame de qualificação. COPPE-UFRJ. (2000)

19. Gray, R. S. \{Agent Tcl\}: A flexible and secure mobile-agent system. In: TCL/TK WORKSHOP, Proceedings... July 1996 (1996) 9-23

20. Aglets - IBM/Japan. Disponível em: $<$ http://www.trl.ibm.com/aglets/>.

21. Straber, M., Baumann J., Hohl, F.: Mole - A Java based Mobile Agent System. In: ECOOP' 96 October (1996) 301-308

22. Junior, I. M., Junior, J. C.: Descoberta e Monitoramento de Recursos em Redes de Computadores usando Agentes Móveis. In: WORKCOMPSUL, Florianópolis (2003)

23. Kendal, E. A. et al.: Patterns of intelligent and mobile agents. In: INTERNATIONAL CONFERENCE ON AUTONOMOUS AGENTS, May (1998) 92-99 